\title{
SEMICLEAN RINGS AND RINGS OF CONTINUOUS FUNCTIONS
}

\author{
NITIN ARORA AND S. KUNDU
}

Dedicated to Professor Larry J. Gerstein

\begin{abstract}
As defined by Ye [12], a ring is semiclean if every element is the sum of a unit and a periodic element. Ahn and Anderson [1] called a ring weakly clean if every element can be written as $u+e$ or $u-e$, where $u$ is a unit and $e$ an idempotent. A weakly clean ring is semiclean. We show the existence of semiclean rings that are not weakly clean. Every semiclean ring is 2-clean. New classes of semiclean subrings of $\mathbf{R}$ and $\mathbf{C}$ are introduced and conditions are given when these rings are clean. Cleanliness and related properties of $C(X, A)$ are studied when $A$ is a dense semiclean subring of $\mathbf{R}$ or $\mathbf{C}$.
\end{abstract}

1. Introduction. All rings will be commutative with identity. As defined by Nicholson, an element $a$ in a ring $R$ is clean $[\mathbf{2 , 7}$ ] if $a$ can be written as $a=u+e$, where $u \in U(R)$, the group of units of $R$, and $e \in \operatorname{Id}(R)$, the set of idempotents of $R$. $R$ is a clean ring if every element is clean. Ye [12] called an element $a$ in a ring $R$ semiclean if $a$ can be written as $a=u+p$, where $u \in U(R)$ and $p \in \operatorname{Per}(R)$, the set of periodic elements of $R$ (that is, $p^{k}=p^{l}$ for $k \neq l$ ). $R$ is called a semiclean ring if every element is semiclean. Several related notions have been studied in the literature, in particular, weakly clean rings [1], $n$-clean rings and $\Sigma$-clean rings $[\mathbf{1 1}]$.

Let $X$ be a completely regular Hausdorff space. Let $1 \in A$ be a subring and subspace of $\mathbf{R}$ in the usual topology, and let $C(X, A)$ denote the set of all continuous $A$-valued functions on $X$. Under pointwise addition and multiplication $C(X, A)$ is a commutative ring with unity.

2010 AMS Mathematics subject classification. Primary 13A99, 13B30, 16S60, $54 \mathrm{C} 40$.

Keywords and phrases. Clean rings, Semiclean, Weakly clean, Rings of continuous functions.

Received by the editors on September 28, 2012, and in revised form on May 5, 2013. 
The subset $C^{*}(X, A)$ of $C(X, A)$, consisting of all bounded functions in $C(X, A)$, is a subring of $C(X, A)$. When $A=\mathbf{R}$, these rings are simply denoted $C(X)$ (likewise $\left.C^{*}(X)\right)[\mathbf{4}]$. We are interested in studying cleanliness and related properties for the rings $C(X, A)$ and $C^{*}(X, A)$ which depend on the topological properties of $X$ and algebraic properties of $A$. Azarpanah [3] and McGovern [6] independently proved that $C(X)$ and $C^{*}(X)$ are clean if and only if $X$ is a strongly zerodimensional space. If $A$ is a proper subfield of $\mathbf{R}$, then $C(X, A)$ is always clean regardless of $X$. Hager and Kimber [5] considered the rings $C(X, A)$ when $A$ is a dense clean subring of $\mathbf{R}$, with unity, which is not a field and $X$ is zero-dimensional. In this case, $C(X, A)$ is clean if and only if $X$ is a $P$-space $[4,4 \mathrm{~J}]$, that is, every $G_{\delta}$-set in $X$ is open (or equivalently, every zero-set is open). We consider the case when $1 \in A$ is a dense semiclean subring of $\mathbf{R}$ or $\mathbf{C}$ which need not be clean.

In Section 2, we show the existence of semiclean rings that are not weakly clean and prove that every semiclean ring is 2-clean. In Section 3 , we introduce classes of dense semiclean subrings of $\mathbf{R}$ and $\mathbf{C}$, and give conditions on when they will be clean. In Section 4, we go on to study cleanliness and related properties of rings of continuous functions, in particular we consider $C(X, A)$, where $A$ is a dense semiclean subring of $\mathbf{R}$. In the final section we study these properties for rings of complex valued continuous functions.

\section{Semiclean rings and other cleanliness related notions.}

Example 2.1. For $p$ prime, denote by $\mathbf{Z}_{(p)}$ the ring $\{(m / n) \in \mathbf{Q} \mid$ $n \notin(p)\}$. It is easy to see that these rings are clean.

In fact, a ring having only trivial idempotents is clean if and only if it is a local ring. In particular, an integral domain is clean if and only if it is local. In [1] an element $a$ in a ring $R$ is called weakly clean if it can be written as $u+e$ or $u-e$, where $u \in U(R)$ and $e \in \operatorname{Id}(R)$. $R$ is called weakly clean if every element is weakly clean. Clearly, every weakly clean ring is semiclean.

Example 2.2. For $p, q$ distinct primes, the $\operatorname{ring} \mathbf{Z}_{(p)} \cap \mathbf{Z}_{(q)}=$ $\{(m / n) \in \mathbf{Q} \mid p \nmid n, q \nmid n\}$ is not clean. For example, $p /(p-q)$ is not a clean element. But these rings are always weakly clean. Suppose 
$m / n$ is not weakly clean. Then each of $m / n,(m / n) \pm 1$ is a non-unit. This means each of $m-n, m$ and $m+n$ is a multiple of either $p$ or $q$. Say, $p$ divides two of them, then $p$ divides $n$, a contradiction.

In [11] an element $a$ is called $n$-clean if it can be written as the sum of $n$ units and an idempotent, and it is $\Sigma$-clean if it is the sum of a finite number of units and an idempotent. Analogously, $n$-clean and $\Sigma$-clean rings are defined. The ring $\mathbf{Z}$ of integers is $\Sigma$-clean but not $n$-clean for any $n$.

Clearly, every clean ring is weakly clean and every weakly clean ring is semiclean. Also every $n$-clean ring is $\Sigma$-clean. Below, we will see that every semiclean ring is 2-clean.

All the examples of semiclean rings that we have seen in literature happen to be weakly clean rings. For example, it was proven in $[\mathbf{1 2}$, Theorem 3.1] that the group rings $\mathbf{Z}_{(p)}(G)$ where $\mathrm{G}$ is a cyclic group of order 3 are semiclean but need not be clean. One can adapt the same proof to prove the following result.

Theorem 2.3. The group rings $\mathbf{Z}_{(p)}(G)$, where $G=\left\{1, a, a^{2}\right\}$ is a cyclic group of order 3 , are weakly clean whenever $p$ is a prime.

Proof. Let us first consider the case $p>3$. In this case the nontrivial idempotents in $\mathbf{Z}_{(p)}(G)$ are $\left(1+a+a^{2}\right) / 3$ and $\left(2-a-a^{2}\right) / 3$ [12, Proposition 3.1]. An element $\left(k+l a+m a^{2}\right) / n, p \nmid n$ in $\mathbf{Z}_{(p)}(G)$ can be expressed as:

$$
\begin{aligned}
0+\frac{k+l a+m a^{2}}{n} & =1+\frac{(k-n)+l a+m a^{2}}{n}=-1+\frac{(k+n)+l a+m a^{2}}{n} \\
& =\frac{1+a+a^{2}}{3}+\frac{(3 k-n)+(3 l-n) a+(3 m-n) a^{2}}{3 n} \\
& =\frac{-1-a-a^{2}}{3}+\frac{(3 k+n)+(3 l+n) a+(3 m+n) a^{2}}{3 n} \\
& =\frac{2-a-a^{2}}{3}+\frac{(3 k-2 n)+(3 l+n) a+(3 m+n) a^{2}}{3 n} \\
& =\frac{-2+a+a^{2}}{3}+\frac{(3 k+2 n)+(3 l-n) a+(3 m-n) a^{2}}{3 n} .
\end{aligned}
$$

We need to show that at least one of the fractional terms to the right of the plus sign is a unit. Suppose not; then, by [12, Corollary 3.1], $p$ 
divides all of:

$$
k^{3}+l^{3}+m^{3}-3 k l m
$$

$$
(k-n)^{3}+l^{3}+m^{3}-3(k-n) l m
$$

$$
(k+n)^{3}+l^{3}+m^{3}-3(k+n) l m
$$

$$
(3 k-n)^{3}+(3 l-n)^{3}+(3 m-n)^{3}-3(3 k-n)(3 l-n)(3 m-n)
$$

$$
(3 k+n)^{3}+(3 l+n)^{3}+(3 m+n)^{3}-3(3 k+n)(3 l+n)(3 m+n)
$$

$$
(3 k-2 n)^{3}+(3 l+n)^{3}+(3 m+n)^{3}-3(3 k-2 n)(3 l+n)(3 m+n)
$$

$$
(3 k+2 n)^{3}+(3 l-n)^{3}+(3 m-n)^{3}-3(3 k+2 n)(3 l-n)(3 m-n) .
$$

As in [12, Theorem 3.1], (2.1), (2.2) and (2.3) above give:

$$
\begin{aligned}
& p \mid\left(-3 k^{2} n+3 k n^{2}-n^{3}+3 n l m\right) \\
& p \mid 3 k .
\end{aligned}
$$

Using (2.9) and the fact that $p \nmid n,(2.8)$ further gives:

$$
p \mid\left(n^{2}-3 l m\right) .
$$

Subtracting (2.4) from (2.7) and using (2.9), we get that $p$ divides

$$
3 n\left(4 n^{2}+n^{2}-2 n^{2}\right)-9 n(3 l-n)(3 m-n)=-27 n(3 l m-l n-m n) .
$$

Using (2.10), $p>3$ and $p \nmid n$ gives:

$$
p \mid(n-l-m) .
$$

Similarly, subtracting (2.6) from (2.5) and using (2.9), we get that $p$ divides

$$
3 n\left(3 n^{2}\right)-9 n(3 l+n)(3 m+n)=-27 n(3 l m+l n+m n) .
$$


Using (2.10), $p>3$ and $p \nmid n$ gives:

$$
p \mid(n+l+m) .
$$

Adding (2.11) and (2.12) gives $p \mid 2 n$, a contradiction. Hence, $p$ does not divide at least one of (2.1)-(2.7). Subsequently, every element of $\mathbf{Z}_{(p)}(G)$ is weakly clean.

When $p=3,(2.1)$ and (2.2) give (2.8) which implies $p \mid n^{3}$, a contradiction. So $\mathbf{Z}_{(3)}(G)$ is clean. When $p=2$, we can argue similar to $\left[\mathbf{1 2}\right.$, Theorem 3.1] to show that $\mathbf{Z}_{(2)}(G)$ is clean.

Is there a semiclean ring which is not weakly clean? The answer is affirmative. But, in order to have this affirmative answer, we need the next lemma.

Lemma 2.4. Let $p_{i}$ be a periodic element in ring $R_{i}: 1 \leq i \leq N$. Then $p=\left(p_{1}, p_{2}, \ldots, p_{N}\right)$ is a periodic element in $R=R_{1} \times R_{2} \times \cdots \times$ $R_{N}$.

Proof. If $p_{i}^{m_{i}}=p_{i}^{n_{i}}\left(m_{i}>n_{i}\right)$, then $p_{i}^{n_{i}}=p_{i}^{m_{i}}=p_{i}^{m_{i}-n_{i}+n_{i}}=$ $p_{i}^{2\left(m_{i}-n_{i}\right)+n_{i}}=\cdots=p_{i}^{k\left(m_{i}-n_{i}\right)+n_{i}}$ for any positive integer $k$. Let $K=\left(m_{1}-n_{1}\right)\left(m_{2}-n_{2}\right) \cdots\left(m_{N}-n_{N}\right), L=\max \left(n_{1}, n_{2}, \ldots, n_{N}\right)$. Then $p^{K+L}=\left(p_{1}^{K+n_{1}+\left(L-n_{1}\right)}, p_{2}^{K+n_{2}+\left(L-n_{2}\right)}, \ldots, p_{N}^{K+n_{N}+\left(L-n_{N}\right)}\right)=$ $\left(p_{1}^{n_{1}+\left(L-n_{1}\right)}, p_{2}^{n_{2}+\left(L-n_{2}\right)}, \ldots, p_{N}^{n_{N}+\left(L-n_{N}\right)}\right)=p^{L}$.

Theorem 2.5. Let $\left\{R_{i}: 1 \leq i \leq N\right\}$ be commutative rings. Then the direct product $R=R_{1} \times R_{2} \times \cdots \times R_{N}$ is semiclean if and only if each $R_{i}$ is semiclean.

Proof. $(\Rightarrow)$. This is clear since every homomorphic image of a semiclean ring is semiclean [12, Lemma 2.1].

$(\Leftarrow)$. Suppose each $R_{i}$ is semiclean. Let $x=\left(x_{i}\right) \in R$. For each $i$, let $x_{i}=u_{i}+p_{i}$, where $u_{i}$ is a unit and $p_{i}$ a periodic element in $R_{i}$. Then $x=u+p$, where $u=\left(u_{i}\right)$ is a unit and $p=\left(p_{i}\right)$ is a periodic element in $R$ by Lemma 2.4 . Hence, $R$ is semiclean.

In particular, a direct product $R=R_{1} \times R_{2}$ of two weakly clean rings is semiclean. However, by [1, Theorem 1.7], $R$ will be weakly clean 
if and only if at least one of $R_{1}$ or $R_{2}$ is clean. This proves that a semiclean ring need not be weakly clean.

Example 2.6. Let $R=\mathbf{Z}_{(3)} \cap \mathbf{Z}_{(5)}$. $\quad((3 / 2),(5 / 2)) \in R \times R$ is not weakly clean. But, subtracting $(1,-1)$ from this gives a unit. By Theorem $2.5, R \times R$ is a semiclean ring.

An element $x$ of a ring $R$ is strongly $\pi$-regular if there exists an $n \in \mathbf{N}$ and $b \in R$ such that $x^{n}=x^{n+1} b$.

Theorem 2.7. Every semiclean ring is 2-clean.

Proof. Clearly, every periodic element is strongly $\pi$-regular and, by [8, Theorem 1], every strongly $\pi$-regular element is strongly clean. In particular, every periodic element is clean. Hence, every semiclean element can be written as the sum of two units and an idempotent.

Again, the subset relation is proper. For example, we will see that $C(X)$ is semiclean if and only if $X$ is strongly zero-dimensional. But every $f \in C(X)$ can be written as the sum of two units (irrespective of $X)$ as $f=(f+|f|+1) / 2+(f-|f|-1) / 2$. So $C(X)$ is 2-clean irrespective of the topology of $X$. For more details on the rings generated by the units, see $[\mathbf{9}, \mathbf{1 0}]$.

Hence, the family of all weakly clean rings is properly contained in the family of all semiclean rings, and the latter family is properly contained in the family of all 2-clean rings.

3. Semiclean subrings of $\mathbf{R}$ and $\mathbf{C}$. Note that the only idempotents in $\mathbf{R}$ are $\{0,1\}$, and the only periodic elements are $\{0,1,-1\}$. Hence, the notions of semiclean and weakly clean coincide for subrings of $\mathbf{R}$. Our conjecture is that this may not be the case with $\mathbf{C}$ which has infinitely many periodic elements.

Below we give more examples of semiclean subrings of $\mathbf{R}$ and $\mathbf{C}$ which need not be clean. We also give the condition when they will be clean.

By $\mathbf{Z}_{(p)}[\sqrt{q}]$, where $p \in \mathbf{N}$ is a prime and $q \in \mathbf{N}$ is not a square, denote the set $\left\{a_{0}+a_{1} \sqrt{q} \mid a_{0}, a_{1} \in \mathbf{Z}_{(p)}\right\}$. Clearly these are subrings of $\mathbf{R}$. 
Also note that an element in these rings can be represented as $\left(k_{0}+k_{1} \sqrt{q}\right) / m, k_{0}, k_{1}, m \in \mathbf{Z}, p \nmid m$.

Lemma 3.1. The element $\left(k_{0}+k_{1} \sqrt{q}\right) / m$ in $\mathbf{Z}_{(p)}[\sqrt{q}]$ is a unit $\Leftrightarrow p$ does not divide $k_{0}^{2}-q k_{1}^{2}$.

Proof. The inverse of $\left(k_{0}+k_{1} \sqrt{q}\right) / m$ in $\mathbf{R}$ is $\left(m\left(k_{0}-k_{1} \sqrt{q}\right)\right) /$ $\left(k_{0}^{2}-q k_{1}^{2}\right)$. This is in $\mathbf{Z}_{(p)}[\sqrt{q}]$ if and only if $p$ does not divide the denominator. (Note that the representation $a_{0}+a_{1} \sqrt{q}$ for a real number with $a_{0}, a_{1} \in \mathbf{Q}$ is unique; otherwise, we can represent $\sqrt{q}$ as a rational.)

Now, $\mathbf{Z}_{(p)}[\sqrt{q}]$ may or may not be clean as shown by the examples below.

Example 3.2. Consider $R=\mathbf{Z}_{(2)}[\sqrt{q}]$. This is a clean ring for all $q$. Suppose $a=\left(k_{0}+k_{1} \sqrt{q}\right) / n \in R$ ( $n$ odd) is such that $a$ and $a-1$ are not units. By Lemma 3.1, $2 \mid k_{0}^{2}-k_{1}^{2} q$ and $2 \mid\left(k_{0}-n\right)^{2}-k_{1}^{2} q$. Subtracting, we get $2 \mid n\left(2 k_{0}-n\right)$. Since $n$ is odd, this is impossible.

Example 3.3. Consider $S=\mathbf{Z}_{(7)}[\sqrt{2}]$. Let $a=(2+3 \sqrt{2}) / 4 \in S$. Clearly, $7 \mid 2^{2}-2 \cdot 3^{2}=-14$ as well as $(-2)^{2}-2 \cdot 3^{2}=-14$. Hence, $a$ and $a-1$ are both non-units. $S$ is not a clean ring.

Theorem 3.4. Let $p \in \mathbf{N}$ be an odd prime, $q \in \mathbf{N}$ is not a square. Let $R$ be the ring $\mathbf{Z}_{(p)}[\sqrt{q}]$. If $p \mid q$, then the ring $R$ is clean. Otherwise, $R$ is clean $\Leftrightarrow q$ is a quadratic nonresidue of $p$.

Proof. Case 1. $p \mid q$. Let $a=\left(k_{0}+k_{1} \sqrt{q}\right) / n, p \nmid n$. Suppose $a$ and $a-1$ are not units. So $p\left|k_{0}^{2}-k_{1}^{2} q \Rightarrow p\right| k_{0}$ and $p \mid\left(k_{0}-n\right)^{2}-k_{1}^{2} q \Rightarrow$ $p \mid k_{0}-n$. Subtracting, we get $p \mid n$, a contradiction. Hence, $R$ is a clean ring.

Case 2. $p \nmid q . \quad(\Rightarrow)$. Suppose $q$ is a quadratic residue of $p$, that is, there exists a $k \in[1, p-1]$ such that $k^{2} \equiv q(\bmod p)$. Take $a=(k+\sqrt{q}) /(2 k) \in R$. Clearly $a$ and $a-1$ are non-units, hence $R$ cannot be clean. 
$(\Leftarrow)$. Suppose $R$ is not clean. Then there exists an $a=\left(k_{0}+k_{1} \sqrt{q}\right) / n$, $p \nmid n$, such that both $a$ and $a-1$ are non-units. So,

$$
\begin{aligned}
& p \mid k_{0}^{2}-q k_{1}^{2} \\
& p \mid\left(k_{0}-n\right)^{2}-q k_{1}^{2} .
\end{aligned}
$$

Subtracting these two, we get $p \mid n\left(2 k_{0}-n\right)$. Since $p \nmid n, p \nmid k_{0}$. Using (3.1), $p \nmid k_{1}$. Hence, there exists a multiplicative inverse $(\bmod p)$ of $k_{1}$. Now, from $(3.1),\left(k_{1}^{-1} k_{0}\right)^{2} \equiv q(\bmod p)$. So, $q$ is a quadratic residue of $p$.

Theorem 3.5. The rings $\mathbf{Z}_{(p)}[\sqrt{q}]$, for $p$ prime, are dense weakly clean subrings of $\mathbf{R}$ which need not be clean.

Proof. We only have to prove that these rings are weakly clean. Suppose, on the contrary, there exists $a=\left(k_{0}+k_{1} \sqrt{q}\right) / n, p \nmid n$, such that none of $a, a-1, a+1$ is a unit. This means

$$
\begin{aligned}
& p \mid k_{0}^{2}-q k_{1}^{2} \\
& p \mid\left(k_{0}-n\right)^{2}-q k_{1}^{2} \\
& p \mid\left(k_{0}+n\right)^{2}-q k_{1}^{2}
\end{aligned}
$$

Subtracting (3.4) from (3.3) and (3.3) from (3.5) gives

$$
\begin{aligned}
& p\left|n\left(2 k_{0}-n\right) \Longrightarrow p\right| 2 k_{0}-n \\
& p\left|n\left(2 k_{0}+n\right) \Longrightarrow p\right| 2 k_{0}+n .
\end{aligned}
$$

Subtracting these two gives $p \mid 2 n$. Since $p \nmid n$, this can happen only if $p$ is 2. But then (3.6) implies $p \mid n$. Again, a contradiction.

Thus, every element $a$ is weakly clean, and hence the $\operatorname{ring} \mathbf{Z}_{(p)}[\sqrt{q}]$ is weakly clean.

Therefore, there are ample examples of dense weakly clean (hence, semiclean) subrings of $\mathbf{R}$ which are not clean. Also, in this case we can find units $u$ in these rings such that $u-1$ and $u+1$ are both non-units. This is because Theorem 3.4 implies we can find $a \in[1, p-1]$ such that $a^{2} \equiv q(\bmod p)$. Then $(p+\sqrt{q}) / a$ is one 
such unit, because $p \nmid p^{2}-q$, but $p \mid(p-a)^{2}-q=p(p-2 a)+a^{2}-q$ and $p \mid(p+a)^{2}-q=p(p+2 a)+a^{2}-q$.

For $p \in \mathbf{N}$, a prime, let us now consider subrings of $\mathbf{C}: \mathbf{Z}_{(p)}[i]=$ $\left\{a+b i \mid a, b \in \mathbf{Z}_{(p)}\right\}$.

We will see that again these rings are weakly clean but need not be clean. Note that there are many more periodic elements in C. So it may be possible to have semiclean subrings of $\mathbf{C}$ which are not weakly clean. For example, one might want to consider rings $\mathbf{Z}_{(p)}[\alpha]$ where $\alpha$ is a $k$ th root of unity.

Lemma 3.6. An element $\left(k_{0}+k_{1} i\right) / m \in \mathbf{Z}_{(p)}[i]$ is a unit $\Leftrightarrow p \nmid$ $k_{0}^{2}+k_{1}^{2}$.

Proof. The inverse in $\mathbf{C}$ of the above element is $m\left(k_{0}-k_{1} i\right) /$ $\left(k_{0}^{2}+k_{1}^{2}\right)$.

Theorem 3.7. For any odd prime $p, \mathbf{Z}_{(p)}[i]$ is a dense weakly clean (hence, semiclean) subring of $\mathbf{C}$, which is clean if and only if $p \equiv 3$ $(\bmod 4)$.

Proof. Suppose $\mathbf{Z}_{(p)}[i]$ is not weakly clean. Then there exists an element $a=\left(k_{0}+k_{1} i\right) / n, p \nmid n$ such that $a, a-1, a+1$ are all nonunits. Therefore, by Lemma 3.6,

$$
\begin{aligned}
& p \mid k_{0}^{2}+k_{1}^{2} \\
& p \mid\left(k_{0}-n\right)^{2}+k_{1}^{2} \\
& p \mid\left(k_{0}+n\right)^{2}+k_{1}^{2} .
\end{aligned}
$$

Subtracting (3.9) from (3.10), $p\left|2 k_{0} \cdot 2 n \Rightarrow p\right| 2 k_{0}$. Subtracting (3.8) from (3.10), $p\left|n\left(2 k_{0}+n\right) \Rightarrow p\right| 2 k_{0}+n$. These two give $p \mid n$, a contradiction.

Hence, every element of $\mathbf{Z}_{(p)}[i]$ has to be weakly clean. However, it need not be clean. For example, consider the element $b=(7+6 i) / 4 \in$ $\mathbf{Z}_{(5)}[i]$. Both $b$ and $b-1$ are non-units in $\mathbf{Z}_{(5)}[i]$ since $3^{2}+6^{2}$ and $7^{2}+6^{2}$ are both multiples of 5 .

As in Theorem 3.4, we can get that $\mathbf{Z}_{(p)}[i]$ is clean $\Leftrightarrow-1$ is a quadratic non-residue of $p$. By Euler's criterion, this will happen whenever $(-1)^{(p-1) / 2} \not \equiv 1(\bmod p)$, that is, $p \equiv 3(\bmod 4)$. 
The rings $\mathbf{Z}_{(2)}[i]$ are clean. The proof is similar to that for the rings $\mathbf{Z}_{(2)}[\sqrt{q}]$.

4. Cleanliness in $C(X, A)$. A non-empty $T_{1}$ space is zerodimensional if it has a base of clopen sets. The rings $C(X)$ are known $[\mathbf{3}, \mathbf{6}]$ to be clean if and only if $X$ is strongly zero dimensional, i.e., the Stone-Čech compactification $\beta X$ is zero-dimensional.

Theorem 4.1. The following are equivalent.

(1) $C(X)$ is clean .

(2) $C(X)$ is weakly clean.

(3) $C(X)$ is semiclean.

Proof. (1) $\Rightarrow(2) \Rightarrow(3)$. Clear.

$(3) \Rightarrow(1)$. This follows from $[\mathbf{6}$, Theorem 13 (iv)] since the periodic elements in $C(X)$ overlap with roots of idempotents. Here we give a direct proof since $[\mathbf{6}]$ had omitted one. Suppose $C(X)$ is semiclean. Let $f$ be any function in $C(X)$. Then $g=2 f-1$ is 1 on $Z(1-f)$ and -1 on $Z(f)$. Since $C(X)$ is semiclean, there exists a periodic function $p$, which assumes one of the values $-1,0,1$, such that $g-p$ is a unit $u$. It is clear that $u<0$ on $Z(f)$ and $u>0$ on $Z(1-f)$. Hence, if we let $K=\{x: u(x)<0\}$, a clopen set, and $\chi_{K}$ be the characteristic function of $K$, then $f-\chi_{K}$ is nowhere zero and hence a unit. So $f$ is clean.

Let $A$ be a dense clean subring of $\mathbf{R}$, with unity, which is not a field. Let $u(A)$ denote the set of its units and $c(A)=\{a \in A \backslash u(A): a-1 \in$ $u(A)\}$. By [5, Lemma 2.2], $u(A)$ and $c(A)$ are dense in $\mathbf{R}$. Further, in [5] it was proved that the rings $C(X, A)$, with $X$ zero-dimensional, are clean if and only if $X$ is a $P$-space.

Is it again the case that $C(X, A)$ is semiclean if and only if it is clean? It certainly is the case when 2 is not a unit in $A$. As in [5], we assume, without loss of generality, that the space $X$ is zero-dimensional. First, we need a couple of lemmas.

Lemma 4.2. Let $A$ be a clean (local) ring with trivial idempotents. Then $A$ has a unit $u$ such that both $u \pm 1$ are non-units if and only if 2 is not a unit in $A$. 
Proof. $(\Leftarrow)$. Take $u=1$.

$(\Rightarrow)$. Let $u$ be such a unit and suppose 2 is a unit. Then $(u \pm 1) / 2$ are both non-units. So $(u+1) / 2$ is not clean, a contradiction.

Lemma 4.3 [5, Lemma 3.6]. Let $X$ be a completely regular Hausdorff space. The following are equivalent.

(1) $X$ is a P-space.

(2) $X$ is zero-dimensional and every countable union of clopen sets is closed.

(3) $f^{-1}(T) \in \operatorname{clop}(X)$ for every $f \in C(X)$ and for every $T \subseteq \mathbf{R}$.

Theorem 4.4. Let $A$ be a dense clean subring of $\mathbf{R}$, with unity, which is not a field, and let $X$ be a zero-dimensional space. If 2 is not a unit in $A$, the following are equivalent.

(1) $X$ is a $P$-space.

(2) $C(X, A)$ is a pm-ring.

(3) $C(X, A)$ is clean.

(4) $C(X, A)$ is semiclean.

Proof. In view of [5, Theorem 3.7], it suffices to prove that $X$ is a $P$-space whenever $C(X, A)$ is semiclean. Let $K_{n}$ be a sequence of pairwise disjoint clopen sets in $X$. Let $u$ be a unit such that $u \pm 1$ are both non-units. Since $c(A)$ is dense in $\mathbf{R}$, let $\left(b_{n}\right)$ be a sequence in $c(A)$ that converges to $u$. Define $f \in C(X, A)$ such that $f \mid K_{n}=b_{n}$ and $f(x)=u$ otherwise. Let $U$ be an open set in $A$. If $u \in U$, then $U$ contains all but finitely many $b_{n}$ 's; thus, $X \backslash f^{-1}(U)$ is closed being a finite union of clopen sets. If $u \notin U$, then $f^{-1}(U)$ is a union of clopen sets and so open. Thus, $f \in C(X, A)$. Since $C(X, A)$ is semiclean, there exist disjoint clopen sets $U$ and $V$ such that $f-\chi_{U}+\chi_{V}$ is a unit of $C(X, A) . \quad f$ is already a unit $(=u)$ outside $\cup_{n} K_{n}$ and adding \pm 1 will make it a non-unit. In $\cup_{n} K_{n}$ it is a non-unit so its value needs to be changed. Hence, $\cup_{n} K_{n}$ has to be $U \cup V$, a clopen set. By Lemma 4.3 (2), it follows that $X$ is a $P$-space.

Now the question arises as to what happens if $2 \in u(A)$. 
Theorem 4.5. Let $A$ be a dense clean subring of $\mathbf{R}$, with unity, which is not a field and such that $2 \in u(A)$. If $X$ is a P-space, then $C^{*}(X, A)$ is a semiclean ring.

Proof. Let $f \in C^{*}(X, A)$. Let $c^{+}(f)=\{x \in X \mid f(x) \geq 0 \in c(A)\}$ and $c^{-}(f)=\{x \in X \mid f(x)<0 \in c(A)\}$. Further, let $u^{+}(A)=$ $\{a \in u(A) \mid a+1 \in u(A)\}$ and $u^{-}(A)=\{a \in u(A) \mid a-1 \in u(A)\}$. Since $2 \in u(A)$, by Lemma $4.2, u^{+}(A) \cup u^{-}(A)=u(A)$. Let $u^{+}(f)=$ $\left\{x \in X \mid f(x) \in(-1 / 2,1 / 2) \cap u^{+}(A)\right\}$ and $u^{-}(f)=\{x \in X \mid f(x) \in$ $\left.(-1 / 2,1 / 2) \cap u^{-}(A)\right\}$. By Lemma $4.3(3)$ (and looking at $f$ as a member of $C(X))$, the sets $c^{+}(f), c^{-}(f), u^{+}(f)$ and $u^{-}(f)$ are all clopen sets and $f-\chi_{c^{-}(f) \cup u^{-}(f)}+\chi_{c^{+}(f) \cup u^{+}(f)}$ is a unit in $C^{*}(X, A)$.

Theorem 4.6. Let $A$ be a dense clean subring of $\mathbf{R}$, with unity, which is not a field. If $X$ is an infinite zero-dimensional space, then $C^{*}(X, A)$ is not a weakly clean ring.

Proof. We can find a sequence $\left\{U_{n}\right\}_{n \in \mathbf{N}}$ of disjoint non-empty clopen sets in $X$, since $X$ is infinite, Hausdorff and zero-dimensional. Let $\left\{a_{n}\right\}_{n \in \mathbf{N}} \subseteq c(A)$ be a sequence such that $a_{n} \rightarrow-1$ and $\left\{b_{n}\right\}_{n \in \mathbf{N}} \subseteq c(A)$ such that $b_{n} \rightarrow 1$. Let $f \in C^{*}(X, A)$ be such that $\left.f\right|_{U_{2 n}}=a_{n}+1$, $\left.f\right|_{U_{2 n-1}}=b_{n}-1$ and $f(x)=0$ otherwise. If $f=u+e$, with $u$ a unit and $e$ an idempotent, then $\left.u\right|_{U_{2 n}}=a_{n}+1$. Since $a_{n}+1 \rightarrow 0$, this makes $u^{-1}$ unbounded, a contradiction. Similarly, $f=u-e$ implies $\left.u\right|_{U_{2 n-1}}=b_{n}-1$, again a contradiction.

Every $P$-space is zero-dimensional [4]. Hence, by Theorems 4.5 and 4.6 , we have a semiclean ring of continuous functions which is not weakly clean.

The above results suggest that if we can find a suitable topological space with lots of clopen sets which is not a $P$-space, we may also be able to demonstrate:

Conjecture 4.7. If 2 is invertible in $A$, a dense clean subring of $\mathbf{R}$, then $C(X, A)$ can be semiclean without being clean.

Let us now consider dense semiclean subrings of $\mathbf{R}$ that are not fields and are not necessarily clean (recall the semiclean rings introduced in 
previous section). Let $S$ denote one such semiclean ring, and consider the ring $C(X, S)$. If $S$ is not clean, then there exists an element $s$ such that both $s$ and $s-1$ are not units. Then the constant function $s$ on any space is not clean. On the other hand, we will see that $C(X, S)$ is semiclean whenever $X$ is a $P$-space. This gives another trivial example of a semiclean ring of continuous functions which is not clean.

Lemma 4.8. Let $S$ be a commutative semiclean ring with unity and only periodic elements $\{-1,0,1\}$. If $S$ is not clean, then $S$ has a unit $u$ such that $u-1$ and $u+1$ are both non-units. If $2 \in u(S)$, then the converse is also true.

Proof. If $S$ is not clean, there exists an $s \in S$ such that $s$ and $s-1$ are both non-units. Hence, $2 s$ and $2 s-2$ are also non-units. Then, $2 s-1$ is the required unit by semicleanliness, since the only periodic elements are $\{-1,0,1\}$.

If $2 \in u(S)$, then by Lemma 4.2 , it follows that $S$ is not clean.

Lemma 4.9. If $S$ is a dense semiclean subring of $\mathbf{R}$ that is not a field, then the set of all non-units in $S$ is also dense in $\mathbf{R}$.

Proof. Let $b$ be a nonzero non-unit in $S$. For every $s \in S, \alpha_{s}=s b$ is also a non-unit. For $r \in \mathbf{R}$, let $\left(s_{n}\right)$ be a sequence in $S$ such that $s_{n} \rightarrow r / b$. Then $\alpha_{s_{n}} \rightarrow r$ shows that the set of non-units is dense in $\mathbf{R}$.

Theorem 4.10. Let $S$ be a dense semiclean subring of $\mathbf{R}$ with unity that is not a field. Let $X$ be a zero-dimensional space.

(1) If $X$ is a $P$-space, then $C(X, S)$ is semiclean.

(2) If $S$ is not clean, then the converse of the above also holds true.

(3) $C(X, S)$ is clean $\Leftrightarrow X$ is a $P$-space and $S$ is clean.

Proof. (1) Let $f \in C(X, S)$. Since we are considering the subspace topology on $S, f$ is also in $C(X)$. Let $c^{+}(f)=\{x \mid f(x) \notin u(S), f(x)+$ $1 \in u(S)\}, c^{-}(f)=\{x \mid f(x) \notin u(S), f(x)-1 \in u(S)\}$. Since $X$ is a $P$-space, by Lemma $4.3(3)$ it follows that $c^{+}(f)$ and $c^{-}(f)$ are clopen sets and $f+\chi_{c^{+}(f)}-\chi_{c^{-}(f) \backslash c^{+}(f)}$ is a unit. Hence, $C(X, S)$ is semiclean. 
(2) If $S$ is not clean, then by Lemma 4.8, there exists a unit $u$ such that $u-1$ and $u+1$ are both non-units. By arguing similarly as in Theorem 4.4 and observing that the set of non-units in $S$ is dense in $\mathbf{R}$, we can now show that the converse holds as well.

(3) If $X$ is a $P$-space and $S$ is clean, then by [5, Theorem 3.7], $C(X, S)$ is clean. Conversely, if $C(X, S)$ is clean then $S$, being a homomorphic image of $C(X, S)$, will be clean. Once again by [5, Theorem 3.7], $X$ has to be a $P$-space.

5. Complex valued continuous functions. In this section we would like to consider the rings of complex valued continuous functions.

For $\mathcal{S}$ a non-empty set of periodic elements of $R$, we say $R$ is $\mathcal{S}$ semiclean if each $x \in R$ can be written as $x=u+p$ where $\mathrm{u}$ is a unit and $p \in \mathcal{S}$.

We begin with proving the following theorem.

Theorem 5.1. The following are equivalent.

(1) $C(X)$ is clean.

(2) $C(X)$ is weakly clean.

(3) $C(X)$ is semiclean.

(4) $C(X, \mathbf{C})$ is clean.

(5) $C(X, \mathbf{C})$ is weakly clean.

(6) $C(X, \mathbf{C})$ is $\mathcal{S}$-semiclean, where $\mathcal{S}$ is the family of all continuous $\{-1,0,1\}$-valued functions.

(7) $X$ is strongly zero-dimensional.

Proof. $(1) \Leftrightarrow(2) \Leftrightarrow(3)$. Theorem 4.1.

$(4) \Rightarrow(5) \Rightarrow(6)$. Clear.

$(6) \Rightarrow(4)$ Similar to $(3) \Rightarrow(1)$ of Theorem 4.1 .

$(4) \Rightarrow(1)$. Given $C(X, \mathbf{C})$ is clean. For $f \in C(X, \mathbf{R}) \subseteq C(X, \mathbf{C})$, let $f=u+e$ where $u=u_{1}+i u_{2}$ is a unit in $C(X, \mathbf{C})$ and $e$ is an idempotent in $C(X, \mathbf{C})$. Now the only idempotents in $\mathbf{C}$ are 0 and 1 , since for a complex $c, c=c^{2}$ implies $c=0$ or $c=1$. So $e$ is real valued, implying $u$ is real valued. Therefore, $f$ is clean in $C(X, \mathbf{R})$ as well. Hence, $C(X, \mathbf{R})$ is clean. 
$(1) \Rightarrow(4)$. Given $C(X, \mathbf{R})$ is clean. Let $f \in C(X, \mathbf{C})$ and $f=f_{1}+i f_{2}$. It is known that $f \in C(X, \mathbf{C}) \Leftrightarrow f_{1}, f_{2} \in C(X, \mathbf{R})$.

From the hypothesis, there exist units $u_{1}, u_{2}$ and idempotents $e_{1}, e_{2}$ in $C(X, \mathbf{R})$, such that $f_{1}=u_{1}+e_{1}$ and $f_{2}=u_{2}+e_{2}$. So $f=$ $u_{1}+e_{1}+i\left(u_{2}+e_{2}\right)$. Now $e_{1}$ is also an idempotent in $C(X, \mathbf{C})$ and $u_{1}$ being non-zero, $u_{1}+i\left(u_{2}+e_{2}\right)$ is a unit in $C(X, \mathbf{C})$. Hence, $f$ is clean and so is $C(X, \mathbf{C})$.

$(1) \Leftrightarrow(7)$. Proved in $[\mathbf{3}, \mathbf{6}]$.

Now we would like to generalize some of the results of $[\mathbf{5}]$ for $C(X, A)$ where $A$ is a subring and subspace of $\mathbf{C}$.

Let $A$ be a dense clean subring of $\mathbf{C}$ with unity, which is not a field. We can generalize Lemma 2.2 of [5] to show that the set $u(A)$ of units in $A$ and $c(A)$, non-units in $A$, are both dense sets in C. Further, Lemma $4.3(3)$ can be generalized so that $f^{-1}(T) \in \operatorname{clop}(X)$ for every $f \in C(X, A)$ and every $T \subseteq \mathbf{C}$. Using these we can show, as in [5, Theorem 3.7] that $C(X, A)$ is clean if and only if $X$ is a $P$-space.

Now, let us consider the case when $S$ is a dense semiclean subring of $\mathbf{C}$ with unity, which is not a field.

Theorem 5.2. Let $S$ be a dense semiclean subring of $\mathbf{C}$ with unity that is not a field. Let $X$ be a zero-dimensional space.

(1) If $X$ is a $P$-space and $|\operatorname{Per}(S)|<\infty$, then $C(X, S)$ is semiclean.

(2) $C(X, S)$ is clean $\Leftrightarrow X$ is a $P$-space and $S$ is clean.

Proof. (1) As in Lemma 4.3 (3), we can show that $f^{-1}(T) \in \operatorname{clop}(X)$ for every $f \in C(X, S)$ and every $T \subseteq \mathcal{C}$ whenever $X$ is a $P$-space. Let $\left\{p_{1}, p_{2}, \ldots, p_{n}\right\}$ be the set of periodics in $S$. Let $c_{0}(S)=u(S)$ and, for $1 \leq i \leq n, c_{i}(S)=\left\{a \in S \mid a-p_{i} \in u(S), a \notin c_{j}(S)\right.$ for $\left.j<i\right\}$. Then $c_{i}(f)=\left\{x \in X \mid f(x) \in c_{i}(S)\right\}$ are clopen sets. Let $p=\Sigma p_{i} \chi_{c_{i}(f)}$. It is easy to show that $p$ is periodic. Then $f-p$ is a unit. Hence $C(X, S)$ is semiclean.

(2) Clear. 


\section{REFERENCES}

1. Myung-Sook Ahn and D.D. Anderson, Weakly clean rings and almost clean rings, Rocky Mountain J. Math. 36 (2006), 783-798.

2. D.D. Anderson and V.P. Camillo, Commutative rings whose elements are a sum of a unit and idempotent, Comm. Algebra 30 (2002), 3327-3336.

3. F. Azarpanah, When is $C(X)$ a clean ring? Acta Math. Hung. 94 (2002), $53-58$.

4. L. Gillman and M. Jerison, Rings of continuous functions, D. Van Nostrand Publ. Co., New York, 1960.

5. A. Hager and C. Kimber, Clean rings of continuous functions, Alg. Univ. 56 (2007), 77-92.

6. W.Wm. McGovern, Clean semiprime $f$-rings with bounded inversion, Comm. Alg. 31 (2003), 3295-3304.

7. W.K. Nicholson, Lifting idempotents and exchange rings, Trans. Amer. Math. Soc. 229 (1977), 269-278.

8. - Strongly clean rings and fitting's lemma, Comm. Alg. 27 (1999), 3583-3592.

9. R. Raphael, Rings which are generated by their units, J. Alg. 28 (1974), 199-205.

10. Peter Vámos, 2-good rings, Q.J. Math. 56 (2005), 417-430.

11. G. Xiao and $\mathrm{W}$. Tong, $n$-clean rings and weakly unit stable range rings, Comm. Alg. 33 (2005), 1501-1517.

12. Y. Ye, Semiclean rings, Comm. Alg. 31 (2003), 5609-5625.

Department of Mathematics, Indian Institute of Technology, Delhi 110016

Email address: nitinarora81@gmail.com

Department of Mathematics, Indian Institute of Technology, Delhi 110016

Email address: skundu@maths.iitd.ac.in 